\title{
Research on Time Division Multi-channel p-Persistent CSMA Based on Binary Trees Conflict Resolution Mechanism
}

\author{
Yifan Zhao, Shengjie Zhou, Hongwei Ding, Qianlin Liu, Zhijun Yang, and Chunfen Li
}

\begin{abstract}
With the rapid growth of wireless communication networks with vary kinds of business, more and more users get a colorful, high-quality information service, which also put forward higher requirements on the quality of service of wireless communication networks. The paper proposed a time division multi-channel p-persistent CSMA based on binary trees conflict resolution mechanism, designed slotted access and combining continuous-time access control clock, using p-persistent probability selection, conducted traffic control by multi-channel priority for random access system. The study use the advantages of integrated slot-type access control and continuous-time access control, with binary trees conflict resolution mechanism which could resolve the conflict and resent the information packets in conflict, such that the system performance has been improved. By modeling analysis, the p-persistent probability can control the protocol throughput. The analytical results and simulation results show that the theoretical analysis and simulation experiments are consistent. At the same time, simulations are also given overview of the probability slotted and time division protocol.
\end{abstract}

Index Terms-Wireless sensor network, p-persistent CSMA, binary tree conflict resolution, multichannel, throughput.

\section{INTRODUCTION}

The wireless sensor network (WSN) is a composed of sensors, data processing Yuan and communication module integrated small network nodes. The nodes form into a dedicated and self-organization wireless network under the control of efficient MAC control protocols and routing protocol of. The sensor nodes can capture the environment temperature, humidity, noise, light intensity, pressure, soil composition, velocity of the moving object, direction and other physical measurements, and then by the channel data processing chip and a dedicated communication circuit point within the acquired collection of information transmit to the control center. Because of this sensor network functional integration, widely used, cost-effective, so it becomes the hot topic inside and outside the country in recent years [1].

With the rapid growth of wireless communication networks with vary kinds of business, more and more users get a colorful, high-quality information service, which also put

Manuscript received November 15, 2015; revised May 12, 2016. This work was supported by the National Natural Science Foundation of China (61461053, 61461054, 61072079); Natural Science Foundation of Yunnan Province (2010CD023); The Financial Support of Yunnan University (No.XT412004).

The authors are with Yunnan University, Kunming, 650091, China (e-mail: 814627093@qq.com, zhoushengjie@hotmail.com, dhw1964@163.com). forward higher requirements on the quality of service of wireless communication networks than the original single QoS. Services business control policy cannot meet the development needs of wireless communication network applications. MIMO, OFDM and other new technologies, but also for multi-channel study MAC control protocol provides condition technology, which is also MAC media access control protocol, and self-organizing nature of the network proposed a higher demand. WSN is the end of the next-generation network will be used, which is in full movement the concept and peer networking technology, the introduction of new network communication protocol to achieve fast networking motion states. In existing random access network communication protocol, it is difficult to meet the mobile self-organization, multi-operational network requirements [2].

In this paper, wireless sensor network, proposes time division multi-channel p-persistent CSMA based on binary trees conflict resolution mechanism, its basic principle is that the channel is the continuous clock manner during channel is idle; the channel is the slot time manner during channel is busy. Upon sensing the channel is idle, at the beginning of the next slot, the nodes send the information packet with probability $\mathrm{p}$, with probability (1-p) abandon send; when the packet idle period that is continuous clock arrives, sent at the same probability $\mathrm{p}$, with probability (1-p) abandon sent [3].

After the introduction of binary collision resolution mechanism, information packet collisions can be sent again in the next time after all the collisions is divided making the system more robust; increases the system throughput [4].

When we introduce the multichannel mechanism, the system has $N$ channels to transmit packets, the nodes occupied of channel resources randomly according to their different business requirements. Each priority has no limit on the number of users, the order of priority from high to low be priority $N$, priority $N-1 \ldots$ priority 1 [5]. Priority $i$ of business occupied the channel 1 to channel $i$, as shown in Fig. 1 . The arrival information packets on the channel $i$ obey Poisson distribution with arriving rate is $G_{i}$, the arrival process of priority $r$ on the channel $i$ obedience the process with arrival rate $\lambda_{i}=G_{i} /(N-i+1)$. Such system is a load balancing system, the same arrival rates for each channel is $G_{i}=G(i=1,2, \ldots, N)$ [6]. Different quality of service according to different requirements, the multi-channel CSMA protocol realized different services of different QoS prioritization requirements by prioritizing. 


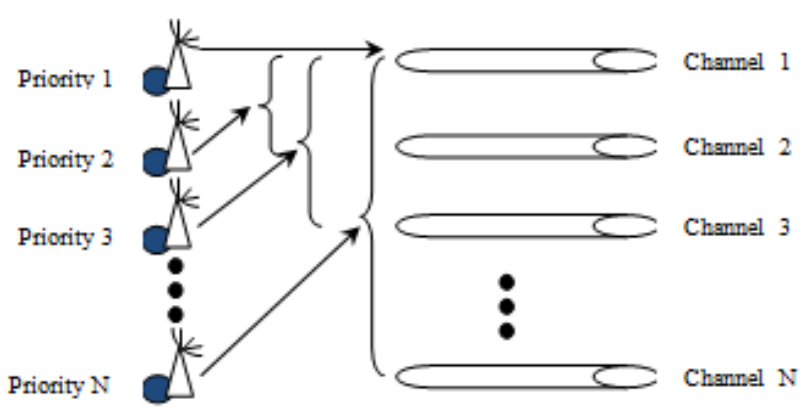

Fig. 1. The system model of multiple channels CSMA.

In this paper, using the averaging cycle period conduct analytical and simulation experiment with the control strategy mentioned above.

\section{THE MODEL}

In the proposed protocol, there will be three random events:

1) $U$ events: Event that information packets are sent successfully.

2) C events: Event that information packets collide with each other (the collision appears).

3) I events: Event that there is no information packets in the channel arrive, the channel is idle.

The model of multichannel three-dimensional probability CSMA protocol with function of monitoring based on binary tree conflict resolution in channel $i$ is showed as Fig. 2 .

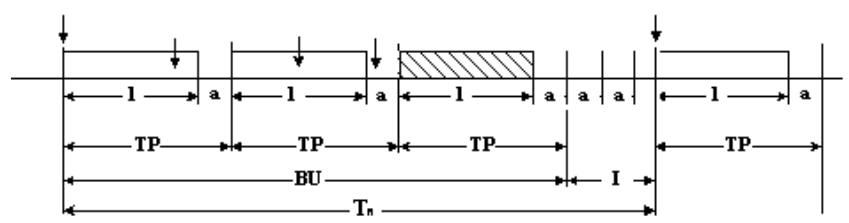

Fig. 2. The model of time division multi-channel p-persistent CSMA based on binary trees conflict resolution mechanism in channel $i$.

Consider the system having $N$ channels and $N$ priorities, the nodes are accessed to the channels randomly by the business priorities of themselves.

Assume that the priority sequence is arranged from low to high as priority 1 , priority $2 \ldots$ priority $N$. The service with priority $i$ occupies channel 1 to $i(i=1,2, \ldots, N)$, that is the service with priority 1 occupies channel 1 , priority 2 occupies channel 1 and channel 2 , and the service with priority $N$ occupies channel 1 to channel $N$ [7].

\section{ANALYSIS OF THE MODEL}

Before analyze the system performance, first do the following assumptions:

1) The channel is ideal with no noise and interference;

2) The basic unit of the system control clock is $a$, the information packets arrived at time $a$ will transmit at the starting time of the next slot [8];

3) The channel propagation delay is $a$, the packet length is unit length and is an integral multiple of $a$;

4) The access method of number $i(i=1,2, \ldots, N)$ channel is timeslot p-persistent CSMA protocol, and the arrival process of number $i$ channel satisfy the Poisson process whose independent parameter is $G_{i}$, each arrival process on the channel is independent of each other [9];

5) The channel using the new protocol, the information packets need to be sent at the first slot in the transmission period can always detecting the state of the channel at last moment;

6) During the transmission of information packets, the phenomenon of packet collisions occur inevitably, and continues to be sent after a random time delay, it sends will not produce any adverse effects on the arrival process channel.

The arrival process of channel satisfies the Poisson process [10]:

$$
P(n)=\frac{(a G)^{n} e^{-a G}}{n !}
$$

In Equation (1), $P(n)$ is the event of $n$ packets arriving during time of $a$.

First, solve the average length $E\left(U_{i}\right)$ of $i$-th channel packet successfully sent in the event of $U$.

Packet successfully sent into the following two cases:

(1) If packets arrive during the last slot of idle period, namely packet arrives at the continuous clock control, and in the next slot time, no one but it adhere to send it, then it is sent successfully, the record for the event is $U i_{1}$.

The average length of $U i_{1}$ in channel $i$ is:

$$
E\left(U i_{1}\right)=E\left(N_{U i_{1}}\right) \times 1=\frac{a p G_{i} e^{-a p G_{i}}}{1-e^{-a p G_{i}}}
$$

(2) If the packet arrives at the busy period, and the packet is the only packet adhere to sent at the current TP period, then the packet will be successfully transmitted within the next TP period, referred to as an event of $U i_{2}$.

At the transmission period, if there is no information packets to be sent in channel $i$, its possibility is:

$$
q_{0}=\sum_{k=0}^{\infty} P\left(A_{k}\right) \times(1-p)^{k}=e^{-p G_{i}(1+a)}
$$

In the transmission period $(1+a)$, if there is only one information packet to be sent in channel $i$, its possibility is:

$$
q_{1}=\sum_{k=1}^{\infty} P\left(A_{k}\right) C_{k}^{1} p(1-p)^{k-1}=p G_{i}(1+a) e^{-p G_{i}(1+a)}
$$

In a cycle, the average length of information packets transmitted successfully at the $U i_{2}$ in channel $i$ is:

$$
E\left(U i_{2}\right)=\frac{q_{1}}{q_{0}}=p G_{i}(1+a)
$$

Then the average length $E\left(U_{i}\right)$ is:

$$
E\left(U_{i}\right)=E\left(U i_{1}\right)+E\left(U i_{2}\right)=\frac{p G_{i} a e^{-p G_{i} a}}{1-e^{-p G_{i} a}}+p G_{i}(1+a)
$$

Secondly, solving average length $E\left(B_{i}\right)$ during the busy period at the $i$-th channel.

$$
E\left(B_{i}\right)=E\left(N_{B_{i}}\right)(1+a)=\frac{1}{q_{0}}(1+a)=\frac{1+a}{e^{-p G_{i}(1+a)}}
$$

Finally, solving average length $E\left(I_{i}\right)$ during the idle period of the $i$-th channel. 
Since the number of idle slots I within the geometric distribution with the mean: $E[N]=\frac{1}{1-e^{-G_{i} p a}}$, an information packet arrive in a time slot with normalized probability: $p_{I 1}=\frac{G_{i} p a e^{-G_{i} p a}}{1-e^{-G_{i} p a}}$, more than an information packet arrives in a time slot with the normalized probability: $p_{I 2}=\frac{1-G_{i} p a e^{-G_{i} p a}-e^{-G_{i} p a}}{1-e^{-G_{i} p a}}$.

Then we get:

$$
E\left(I_{i}\right)=\left(\frac{1}{1-e^{-G_{i} p a}}-1\right) a+\frac{G_{i} p a^{2} e^{-G_{i} p a}}{2\left(1-e^{-G_{i} p a}\right)}+\frac{\left(1-G_{i} p a e^{-G_{i} p a}-e^{-G_{i} p a}\right) a}{1-e^{-G_{i} p a}}
$$

The throughput of the new protocol in channel $i$ is:

$$
\begin{aligned}
S_{i} & =\frac{E\left(U_{i}\right)}{E\left(B_{i}\right)+E\left(I_{i}\right)} \\
& =\frac{\frac{p G_{i} a e^{-p G_{i} a}}{1-e^{-p G_{i} a}}+p G_{i}(1+a)}{\frac{1+a}{e^{-p G_{i}(1+a)}}+\left(\frac{1}{1-e^{-G_{i} p a}}-1\right) a+\frac{G_{i} p a^{2} e^{-G_{i} p a}}{2\left(1-e^{-G_{i} p a}\right)}+\frac{\left(1-G_{i} p a e^{-G_{i} p a}-e^{-G_{i} p a}\right) a}{1-e^{-G_{i} p a}}}
\end{aligned}
$$

When there's more than one packet arrive, the collision will happen and the conflict will be resolved at the next time slot according to the modified binary tree conflict resolution mechanism. When there are some packets arrive in the conflict resolution, they will be transmitted according to the non-persist CSMA protocol. After the resolution is done, the new packets will be transmitted according to the new protocol.

In a cycle, for channel $i$, the average effective length that collision packets have been successfully divided and retransmitted is:

$$
\begin{aligned}
E\left(N_{B x_{i}}\right) & =\sum_{x=2}^{\infty} \frac{\left[p G_{i}(1+a)\right]^{x} e^{-p G_{i}(1+a)}}{x !\left(1-e^{-a p G_{i}}\right) e^{-a p G_{i}}} \\
E\left(U_{B x_{i}}\right) & =E\left(N_{B x_{i}}\right) \times x \\
& =\sum_{x=2}^{\infty} \frac{x\left[p G_{i}(1+a)\right]^{x} e^{-p G_{i}(1+a)}}{x !\left(1-e^{-a p G_{i}}\right) e^{-a p G_{i}}}
\end{aligned}
$$

The average length $E\left(U_{i}^{*}\right)$ is:

$$
\begin{aligned}
E\left(U_{i}^{*}\right) & =E\left(N_{U_{i}}\right) \times(1+a) \\
& =\frac{q_{1}}{q_{0}\left(1-q_{0}\right)} \times(1+a) \\
& =\frac{(1+a) p G_{i}(1+a) e^{-p G_{i}(1+a)}}{e^{-p G_{i} a}\left(1-e^{-p G_{i} a}\right)}
\end{aligned}
$$

If there are $x$ collision packets, they will choose the right or left time slot separately and randomly. Assuming its probability is $p_{i j} . p^{\prime}{ }_{i j}$ is the probability that $i$ packets come to the left time slot and $j$ packets come to the right time slot. $p^{\prime}{ }_{i j}$ is the probability that $j$ packets come to the left time slot and $i$ packets come to the right time slot.

$$
\begin{gathered}
p_{i j}=p_{i j}^{\prime}+p_{j i}^{\prime} \\
p_{i(x-i)}=2 ! C_{x}^{i} C_{x-i}^{x-i} p^{x}=2 ! C_{x}^{i} p^{x}
\end{gathered}
$$

In (14), $x$ is the odd number, $i=0,1, \ldots,[N / 2]$.

$$
p_{(x / 2)(x / 2)}=C_{x}^{x / 2} p^{x}
$$

In (15), $x$ is the even number.

The average length of successfully resolve the $x$ collision packets are:

$$
\begin{aligned}
E\left(L_{x}\right)= & p_{0 x}\left[1+E\left(L_{x}\right)\right]+p_{1(x-1)}^{\prime}\left[1+E\left(L_{x-1}\right)\right] \\
& +p_{(x-1) !}^{\prime}\left[2+E\left(L_{x-1}\right)\right]+\sum_{i=2}^{[x / 2]} p_{i(x-i)}\left[1+E\left(L_{i}\right)+E\left(L_{x-i}\right)\right] \\
= & \frac{p_{0 N}+p_{1(x-1)}^{\prime}\left[3+2 E\left(L_{x-1}\right)\right]}{1-p_{0 x}}+\frac{\sum_{i=2}^{[x / 2]} p_{i(x-i)}\left[1+E\left(L_{i}\right)+E\left(L_{x-i}\right)\right]}{1-p_{0 x}} \\
E\left(B x_{i}\right) & =E\left(N_{B x_{i}}\right)(1+a) \times\left(x+E\left(L_{x}\right)\right) \\
& =\sum_{x=2}^{\infty} \frac{\left[p G_{i}(1+a)\right]^{x} e^{-p G_{i}(1+a)}(1+a)}{x !\left(1-e^{-a p G_{i}}\right) e^{-a p G_{i}}} \bullet\left(x+E\left(L_{x}\right)\right)
\end{aligned}
$$

The throughput of time division multi-channel p-persistent CSMA based on binary trees conflict resolution mechanism for channel $i$, is:

$$
S_{i}^{\prime}=\frac{E\left(U_{i}\right)+E\left(U_{B x_{i}}\right)}{E\left(U_{i}^{*}\right)+E\left(I_{i}\right)+E\left(B x_{i}\right)}
$$

In the $N$ channels of wireless communication system, because this channel model is a load equilibrium model, so the arrival probabilities of each channel are the same, that is to say:

$$
G_{1}=G_{2}=G_{3}=\cdots=G_{i}=\cdots=G_{N}=G
$$

Basing on the above analysis and computational formula of the systemic throughput.

The systemic throughput of time division multi-channel p-persistent CSMA based on binary trees conflict resolution mechanism is:

$$
S=N S_{i}^{\prime}
$$

The arrival probability of the business with the priority $l$ in channel $i$ is

$$
\lambda_{i}=\frac{G_{i}}{N-i+1}(i \leq l)
$$

Assuming that the length of information packet sent by the business with priority $l$ successfully in the average cycle period of channel $i$ is: $E\left(U_{i}^{(p l)}\right)(i \leq l)$.

Then according to the above analysis, we can get the throughput of the time division multi-channel p-persistent CSMA based on binary trees conflict resolution mechanism with the priority $l$ :

$$
S_{p l}=\left(\sum_{i=1}^{l} \frac{1}{N-i+1}\right) S_{i}^{\prime}
$$

\section{Simulation}

From the above analysis, the expression of the system 
throughput under the time division multi-channel p-persistent CSMA based on binary trees conflict resolution mechanism is got. Based on the above analysis, with the use of simulation tool: MATLAB R2010a, the simulation results are shown as following. During the simulation, transmission delay time: $a=0.01$.
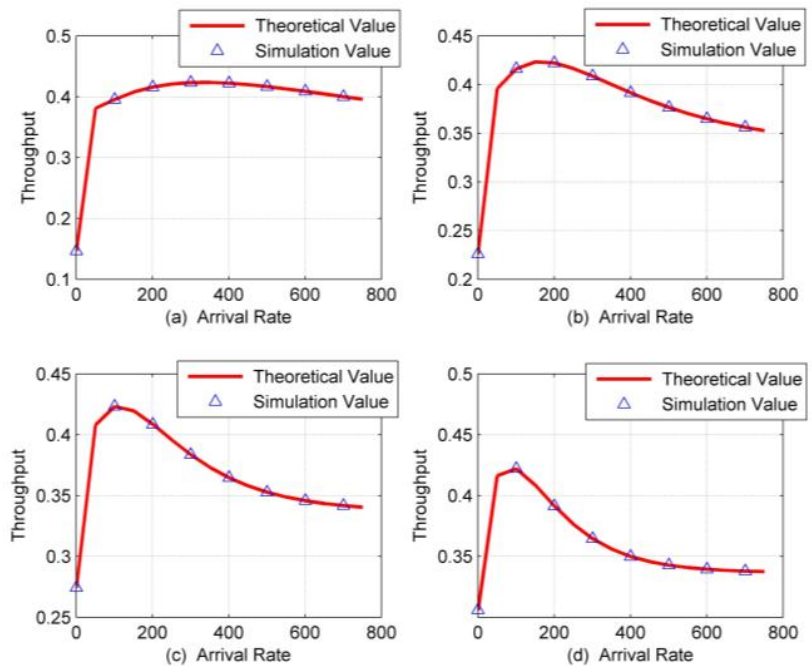

Fig. 3. The throughput of the new protocol with different $p($ a) $p=0.2$; (b) $p=0.4$; (c) $p=0.6$; (d) $p=0.8$ ) for channel $i$.

In Fig. 3, the simulation values of system throughput under the new protocol are consistent with the theoretical ones, verified the correctness of mathematical derivation done before. When $G_{i}$ increase greatly, the system throughput holds a value stably. It means that the new protocol can adapt to variety of different network environment, heavy network load in particular because the introduction of binary trees conflict resolution. In addition, the new protocol can perform better than other protocols when the channel arrival rate $G_{i}$ is great. Also we can see that we are able to control the system throughput by change the value of probability $p$.

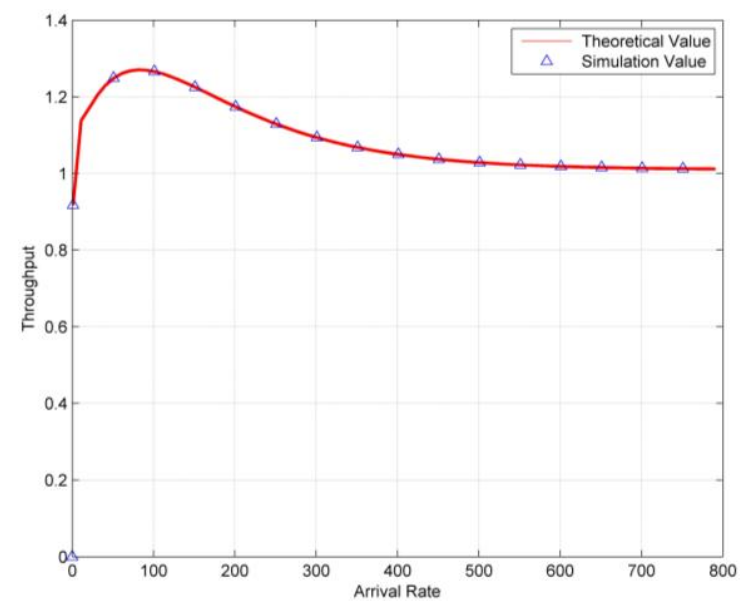

Fig. 4. The throughput of the new protocol with 3 channels $(p=0.8)$.

Fig. 4 through Fig. 7, the simulation values of system throughput under the new protocol are consistent with the theoretical ones, verified the correctness of mathematical derivation done before. With the number of channels increases, the new protocol's total system throughput increases. By the adoption of the multi-channel mechanism we can distribute the channel resources to every channel according to their priority separately; it means that the priority is higher, the corresponding single channel can get more network resources; the higher priority channel is, the more network resources it gets.

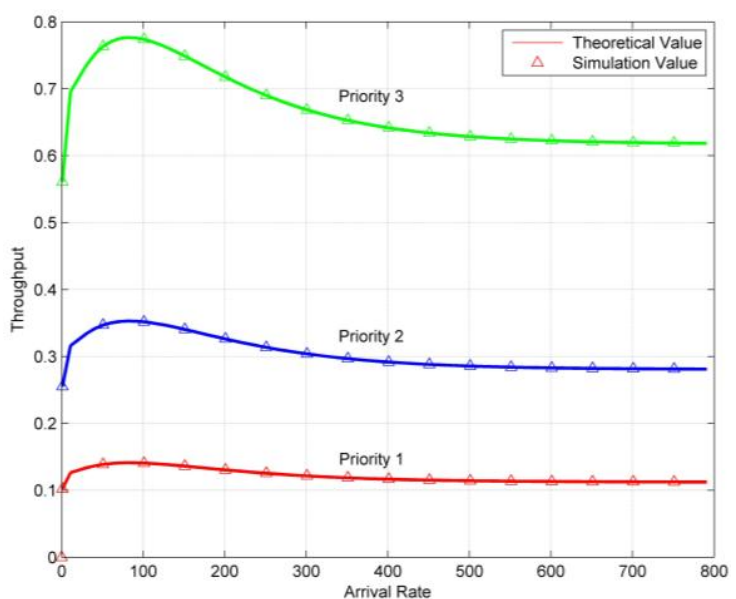

Fig. 5. The comparison of 3 channels with different priorities $(p=0.8)$.

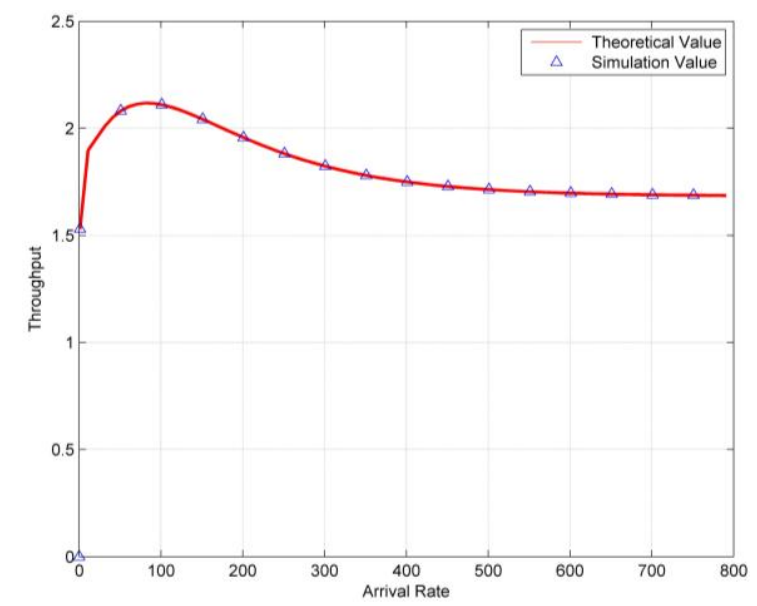

Fig. 6. The throughput of the new protocol with 5channels $(p=0.8)$.

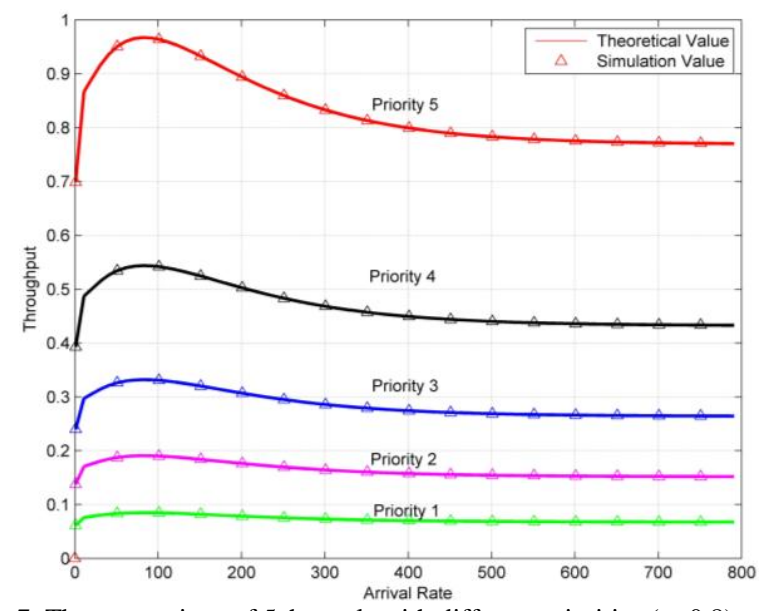

Fig. 7. The comparison of 5 channels with different priorities $(p=0.8)$.

\section{CONCLUSIONS}

The time division multi-channel P-persistent CSMA based on binary trees conflict resolution mechanism, using the average cycle method, gets the precise mathematical expressions of system throughput by rigorous mathematical derivation, designed slotted access and combining continuous-time access control clock, using p-persistent 
probability selection, conducted traffic control by multi-channel priority for random access system. With binary trees conflict resolution mechanism, the system could resolve the conflict and resent the information packets in conflict one by one, such that the system performance has been improved. By modeling analysis, the P-persistent probability can control the protocol throughput. The analytical results and simulation results show that the theoretical analysis and simulation experiments are consistent. The proposed protocol improves the controllability of the system, the channel utilization, system security, and reliability of packet transmission, meets the different priority of different QoS requirements.

\section{REFERENCES}

[1] D. F. Zhao, "Study on A new method for continuous-time systems of random access channel," Journal of Electronics, vol. 21, no. 1, pp. 37-41, 1999

[2] H. W. Ding, Y. Y. Guo, Y. F. Zhao, S. J. Zhou, and Q. L. Liu, "Research on the multi-channel probability detection CSMA protocol with sensor monitoring function," Sensor Lett., vol. 13, pp. 143-146, 2015.

[3] S. J. Zhou et al., "Research on the discrete time three-dimensional probability CSMA protocol in Ad-Hoc network," International Journal of Recent Scientific Research, vol. 6, issue 5, pp. 4257-4262, June 2015

[4] D. F. Zhao, B. H. Li, and S. M. Zheng, "Study on a new method for the slotted access channel," Journal of Electronics, vol. 19, no. 6, pp. 814-819, 1997.

[5] Y. Shang and H. C. Shi, "Flexible energy efficient density control on wireless sensor networks," International Journal of Distributed Sensor Networks, vol. 3, no. 1, pp. 101-120, 2007.

[6] B. B. Liu, "The analysis of multi-channel random multiple access wireless communication network protocol based on probability detection,” Kunming: Yunnan University, 2006, pp. 55-59.
[7] Z. C. Ma, Y. N. Sun, and T. Mei, "Survey on wireless sensor network," Journal of China Institute of Communications, vol. 25, no. 4, pp. 114-124, 2004.

[8] J. C. Huang, H. Xie, and B. Z. Xu, "Random prediction tree protocol decomposing collision packets," Journal of China Institute of Communications, vol. 3, p. 21, 1983.

[9] D. F. Zhao, "Study on the average cycle method for slotted multiple-access communications," Journal of China Institute of Communications, vol. 20, no. 8, pp. 80-85, 1999.

[10] D. F. Zhao, "Study on A new method for the slotted access channel," Journal of Electronics, vol. 19, no. 6, pp. 814-819, 1997.

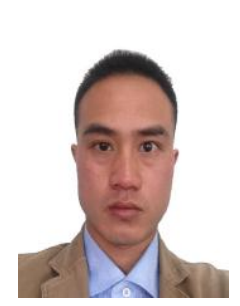

Shengjie Zhou was born in Yunlin, Guangxi in February 1988. He received the bachelor of engineering degree from Yunnan University, Kunming, China, in 2011. Currently, he is a graduate student at Yunnan University. His major field of research is random multiple access.

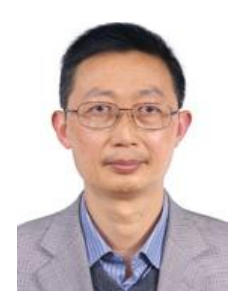

Hongwei Ding was born in 1964. He chaired or participated in more than 20 projects. Among them, presided over 10 research projects, including National fund, Provincial fund, Department of Education funds, school funds and other six large projects in Yunnan, as well as four horizontal projects; he is a member of 10 major subjects of research. Among the 20 topics, there are national issues six provincial fund, a Department of Education Fund, a fund of Yunnan University and horizontal project. He obtained a Yunnan Natural Science Award, in December 2013; published more than 100 papers, including the first author or corresponding author SCI, EI retrieval; access to software copyrigh registration of more than 20 items. Currently, his research interests include random multiple access system, polling multiple access systems and wireless sensor networks. 